\title{
Smart Parking Assistance Services and User Acceptance: A European Model
}

\begin{abstract}
Keywords: parking sensors, parking assistance, survey, user constraints, user acceptance
Abstract: $\quad$ Technologies and systems assisting drivers to locate free on street parking space and/or inform on parking availability may significantly reduce the traffic induced from cruising for parking space in cities. This paper attempts to reveal the factors that may affect the acceptability of parking assistance systems in different European cities, based on data collected through a questionnaire survey. The respondents are presented with a real world parking assistance system based on in-vehicle ultrasonic sensors, which detects free parking space in real time, and are, then, asked to respond to a set of questions in relation to their parking choice preferences. The results of the survey are presented and modelled using a genetically optimized Logistic Regression Model. Findings indicate that the proposed system would be useful for people who are not willing to spend too much time in order to find an available parking space as well as to those who are not willing to walk long distances from the parking place to their final destination. Moreover, results revealed that the certainty of the provided recommendation significantly influences the effect of the other parameters on the acceptability of the application. Finally, some further research steps are discussed.
\end{abstract}

\section{INTRODUCTION}

With the increase in demand for traveling in the cities, the demand for parking areas increases leading drives to circulate inside urban area (cruising) in search for a parking space. Cruising for a parking space leads to increased fuel consumption and induced traffic congestion (Shoup, 2006, Arnott \& Inci, 2006). Shoup (2006) found in specific time periods of a day cruising for parking space may account for $50 \%$ of the traffic. Van Ommeren et al. (2011) estimated that cruising for parking may increase commuting by approximately $20 \%$.

In order to tackle the negative impacts of cruising for a parking space, several attempts based on theoretical urban economics to regulate parking prices in and out of city centres (Arnott \& Rowse, 1999) have been conducted. Several studies investigated the effect of parking policies on traffic management using simulation of real world data (Shiftan \& Burd-Eden, 2001, Chatman \& Manville, 2014).

Literature for long has indicated that the time spent by drivers searching for a parking space is related to the availability of parking information (Ahangari et al., 2018). This availability is related to: i. real time parking space monitoring infrastructure, ii. the ability to produce crowdsourced information on free street parking space and iii. the provision of advanced parking assistance systems. Some prominent examples of monitoring parking spaces using underground magnetic sensors, such as the FASTPRK project in Moscow (50000 sensors) (Worldsensing, 2019) and SFpark project in San Francisco (8200 sensors in 2014) (Chatman \& Manville, 2014). A dynamic approach, entailing the collection of data from a moving unit either vehicle or drone can be also a potential solution (D'Aloia et al., 2015, Golias \& Vlahogianni, 2018). These approaches are established on deep learning and other advanced algorithms for parking space identification and availability prediction (Vlahogianni et al., 2015, Monteiro \& Ioannou, 2018, Golias \& Vlahogianni, 2018).

Various parking assistance systems have been developed and used worldwide in order to reduce the existing parking problems and to improve the efficient use of the existing parking supply. Smart parking systems are already used for defining parking occupancy, parking guidance information and, parking facility management while different technologies are used for parking space detection like inductive loops, infrared sensors, magnetometers, ultrasonic detectors, radar sensors, etc. (Revathi \& Dhulipala, 2012, Fraifer \& Fernström, 2016, Faheem et al., 2013, Lin et al., 2017). All the parking systems aim at assisting drivers while cruising for parking and make the parking search less time consuming and easier.

But, how influential is the provision of parking information to the users' parking behaviour? Past studies have underlined that the time seeking for a 
parking space appears to be critical in the parking choice behaviour (Ibeas et al., 2014). Soto et al. (2018) found that, in addition to parking fee, search time and access time, a risk-averse attitude and a positive car care (maintenance) attitude are determinants for parking choice. The importance of parking assistance systems has been studied in Ahangari et al. (2018) using a stated preference survey and a driving simulator to evaluate the effects of different types of information related to parking space availability on parking choice and circulation behaviour. The study revealed that age and parking availability information affect parking choice behaviour.

The scope of this paper is to evaluate the acceptance of parking assistance systems in a European level based on a questionnaire survey. The survey is based on a real-world novel parking assistance technology based on ultrasonic sensors installed in the vehicle, which detects free parking spaces and provides information to the drivers about parking space availability near their destination. The survey is conducted in order to reveal users' expectations and needs from such a tool and also to present and analyse their willingness to use it. Finally a genetically optimized Logistic Regression Model is applied in order to reveal the factors affecting users' acceptability of such a parking assistance tool and their willingness to integrate it in their everyday life.

\section{ACCEPTANCE OF SMART PARKING ASSISTANCE SYSTEMS}

\subsection{The Reference System}

The proposed parking assistance system uses ultrasonic sensors installed in the vehicle and enables the detection of free gaps on the right and left side of a street when an equipped vehicle passes by. The ultrasonic system is able to detect the complete scene with its limiting vehicles, curb stone information, length and depth of the detected gap and other additional attributes. The concept of the proposed technology is illustrated in Figure 1.

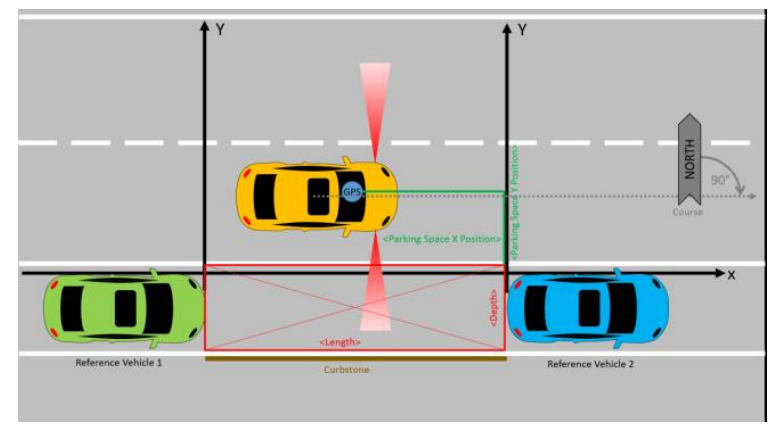

Figure 1: Ultrasonic parking place detection principle.

The data is transmitted to a backend server and based on historic information and a parking area map the detected gap is classified either as a parking space or a non-parking space (driveways, exits, etc.). More specifically, the parking place detections resulting out of driveways etc. are filtered out whereas other parking places are validated as real parking spaces where parking is indeed allowed (Margreiter et al., 2017). The proposed technology aims not only at detecting free parking spots but also at predicting parking space availability around the destination of the driver and for a certain arrival time. This challenge is solved by using historic occupancy data which is updated by real time data collected from available transmitter vehicles in the region. Through this system the users can be informed about free parking space availability and existence at any time near their destination and be guided towards the free parking spot without having to drive around the same blocks. More details about the technology and its services can be found in Margreiter et al. (2015), Margreiter et al. (2017) and Orfanou et al. (2017).

\subsection{The Survey}

Beside the continuously increase of smart parking assistance systems, the success of such a system is based on its acceptance from the potential users (drivers). For this reason, a survey was conducted to get more detailed information about driver's parking behaviour and habits. Moreover, the respondents had to give answers related to their needs and their expectations from a parking assistance system as well as if and under which circumstances they are willing to accept and use such a system.

The National Technical University of Athens and the Technical University of Munich conducted a survey in order to investigate how willing the drivers are to use such an application described in the previous section. The two research teams collected 
more than 500 answers and the respondents were coming from different European cities developing a cultural and habitual diversity among the participants. Due to the fact that parking assistance technologies should address the needs of drivers coming from different countries and cities it is important to detect the differences in their parking habits, needs and constraints so that the system can meet and satisfy the expectations of all potential users.

The distributed questionnaire was divided in three parts. In the first part, the participants had to give answers related to their parking behaviour. In the second part, an overview and brief description of the new developed technology was given and the potential users revealed their expectations from such a technology, as well as their willingness to use it. The last part contained some personal information about the gender and age of the respondents.

The age and gender distribution in the sample is presented in Figures 2 and 3. Overall, 66\% of the responders are men and $34 \%$ women, while the majority of them belong to the age group 26-35 years old.

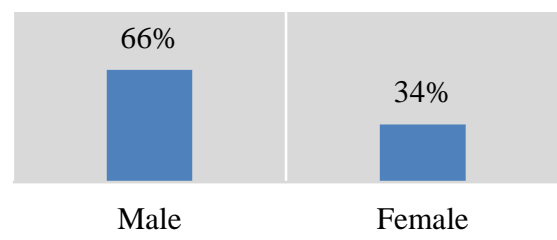

Figure 2: Gender distribution in the sample

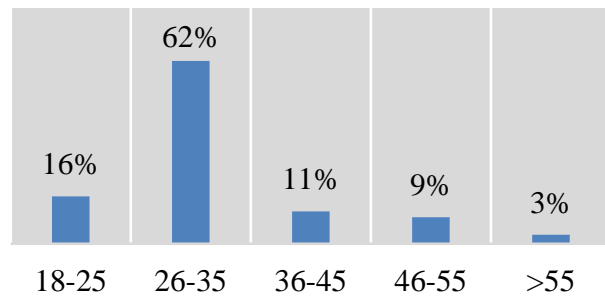

Figure 3: Age distribution in the sample

As mentioned before, here it is attempted to analyse parking behaviour and the willingness to use a parking assistance system of people living in several European cities. To this end, the sample includes citizens of the biggest cities of Greece, Germany, Austria and Switzerland, while there are some respondents from Italy, Portugal and Cyprus but they are underrepresented. In addition, several characteristics of each city were included with their corresponding levels as shown in the Table 1.
Table 1: City attributes and the corresponding levels

\begin{tabular}{|l|c|c|c|}
\hline Attributes & \multicolumn{3}{|c|}{ Levels } \\
\hline Population & $<100000$ & $<500000$ & $>500000$ \\
\hline Area & $<50 \mathrm{~km}^{2}$ & $<200 \mathrm{~km}^{2}$ & $>200 \mathrm{~km}^{2}$ \\
\hline $\begin{array}{l}\text { Access to } \\
\text { the internet }\end{array}$ & $\begin{array}{c}\text { High } \\
(>75 \%)\end{array}$ & $\begin{array}{c}\text { Medium } \\
(>50 \%)\end{array}$ & $\begin{array}{c}\text { Low } \\
(<50 \%)\end{array}$ \\
\hline $\begin{array}{l}\text { Usage of } \\
\text { goods and } \\
\text { services } \\
\text { that are } \\
\text { obtained } \\
\text { through the } \\
\text { internet }\end{array}$ & $\begin{array}{c}\text { High } \\
(>75 \%)\end{array}$ & $\begin{array}{c}\text { Medium } \\
(>50 \%)\end{array}$ & $\begin{array}{c}\text { Low } \\
(<50 \%)\end{array}$ \\
\hline $\begin{array}{l}\text { Frequency } \\
\text { of internet } \\
\text { use }\end{array}$ & $\begin{array}{c}\text { High } \\
(>75 \%)\end{array}$ & $\begin{array}{c}\text { Medium } \\
(>50 \%)\end{array}$ & $\begin{array}{c}\text { Low } \\
(<50 \%)\end{array}$ \\
\hline
\end{tabular}

Data processing procedures included the exclusion of incomplete questionnaires as well as other fault or malicious answers, such as household size greater than 20 . The final sample size includes a total number of 374 questionnaires.

\section{FINDINGS}

\subsection{Preliminary Assessment of Responses}

A preliminary assessment of the responses was conducted first. Results showed that $51 \%$ of the sample spent less than 5 minutes to find a free parking space while only $4 \%$ of the sample have to spend more than 20 minutes. As far as it concerns the time drivers are willing to spend on finding a free parking space results are almost the same, as shown in Figure 4.

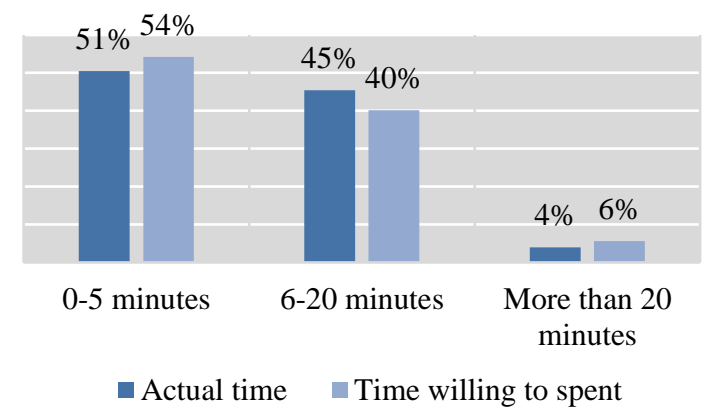

Figure 4: Time spent and time drivers are willing to spend on finding a free parking space

Moreover, the vast majority of the sample are not willing to walk more than $1 \mathrm{~km}$ from their car to their final destination, while in addition $54 \%$ of the sample stated that their tolerable distance between the 
parking place and their final destination is less than 400m.

Furthermore, most of the drivers $(70 \%)$ prefer to park on the street, rather than to park in a public $(18 \%)$ or private $(12 \%)$ parking garage. By means of parking strategies, most of the drivers $(74 \%)$ prefer to search for a free parking space between several city blocks while only $19 \%$ of the drivers try to find a parking space by driving within the same block. The rest of the sample prefers to either wait passively at the same place for a space to be free or drive only along a particular road with intermediate reversals. These results also highlight the need for an application which provides information on the availability of parking places on the street as the one described in the present work.

Figure 5 presents the importance of several aspects of the proposed application as they were rated by the respondents. Findings revealed that the two most important aspects of the application for the majority of the sample are the provision of information about the type of the available parking place (e.g. place for habitants only, place for people with disabilities) and the saving of time when searching for a parking space. In addition, of great importance is the depiction of the exact location of the available parking space as well as the ability of the app to recommend the parking places which are close enough to the final destination. On the other hand, results revealed that the least important aspect of the application is the ability to book a parking space on the road.

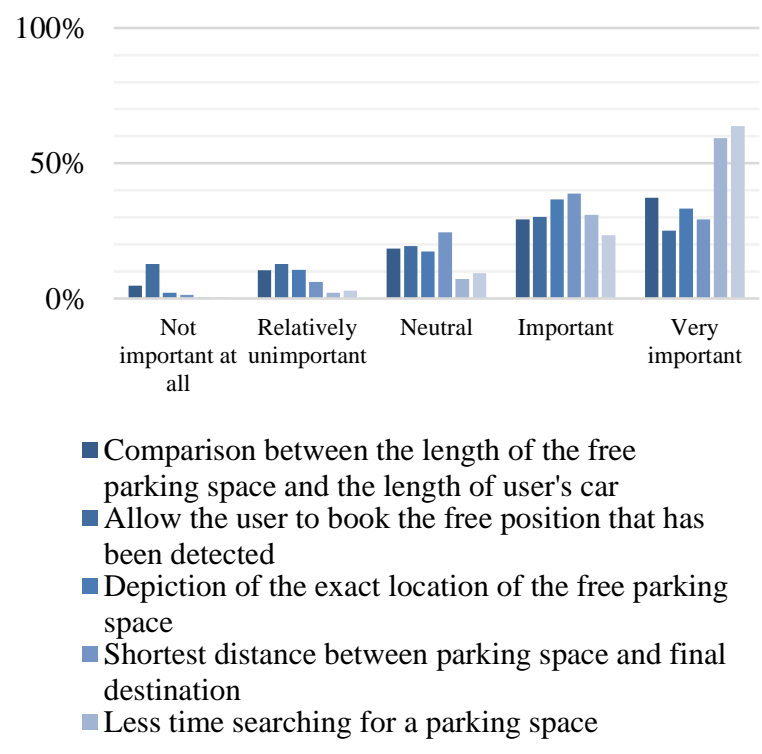

Figure 5: Distribution of answers concerning the importance of the aspects of the application
Interestingly, only $23 \%$ of the drivers require that the information on the availability of parking is at least $90 \%$ certain in order to be provided through the application, while the rest of them would like to receive this information although may be not so valid. More specifically, almost $60 \%$ of the sample wants to receive this information when this information is 70 - $90 \%$ valid that the detected gap corresponds to a free parking space instead of an exit or a driveway.

Finally, respondents were asked to select between three alternatives concerning the kind of information that they want to receive. In terms of the recommendation of a free parking space the application may:

1. Suggest an eventually longer route to the destination with a higher availability of free parking places (suggestion of the appropriate route).

2. Depict the availability of free parking places for all city blocks in the area around the destination.

3. Recommend one specific free parking place close to the destination.

The corresponding results are shown in Figure 6. The responses show that users will prefer the much more realistic alternative for the system, which is to depict the availability of free parking places for all city blocks in the area around the destination. This may be due to the fact that they are used to rely on experience and do not necessarily need the system to point to a specific free parking space.

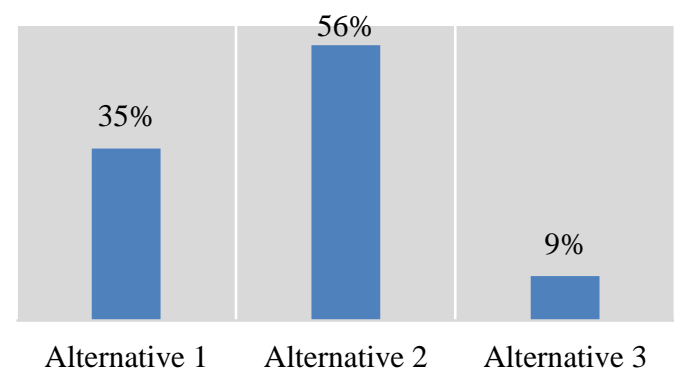

Figure 6: Distribution of the sample between the three alternatives concerning the type of provided information on the availability of parking spaces.

\subsection{A European Model of Parking Assistance Systems Acceptance}

In this section, the most significant variables that affect the acceptance of the proposed system are being discussed. For this purpose, a Genetically Optimized Logistic Regression model was developed taking into consideration the interactions between 
independent variables. The search for the best model, especially in cases where there are many predictors to be considered of different form, is exhaustive and time consuming. Using nature-inspired metaheuristics, such as genetic algorithms, swarm optimization etc., for big size problems makes the searching for the near optimum solution much more computationally efficient and flexible when compared to exact optimization algorithms (Vlahogianni et al., 2014). In this paper, the genetically based searching algorithm of Calcagno \& de Mazancourt (2010) is implemented, which is based on the Yang's enhanced genetic search operator with an immigration function to improve convergence for complex problems (Yang, 2004).

The dependent variable in the model is a binary variable describing the acceptability of the system, taking the value 1 if the respondent is willing to use the application and the value 0 otherwise. As independent variables are used the demographics of the users (gender, age, car ownership) as well as drivers' habits and strategies when searching for a free parking space. Regarding the search algorithm, the fitness function $w_{i}$ for the $\mathrm{i}^{\text {th }}$ model is given by:

$$
w_{i}=e^{\left(-\left(Q A I C c_{i}-Q A I C c_{\text {best }}\right)\right)}
$$

where $\mathrm{QAICc}_{\mathrm{i}}$ is the information metric for the $\mathrm{i}^{\text {th }}$ model and $\mathrm{QAIC} c_{\text {best }}$ the information metric for the best model in the population of models (Burnham \& Anderson, 2002). As a note, higher $\mathrm{QAICc}_{\mathrm{i}}$ means lower fit.

Results are summarized in the table below (Table 2). The accuracy of the model is $76 \%$, while the precision is $73.7 \%$.

The estimated coefficients of the regression model indicate to what extent the acceptance of the application changes when different parameters (independent variables) change. In the case where pairwise interactions are being considered, the estimated coefficients describe the simultaneous influence of the two variables to the dependent variable.

According to the results, male drivers are more likely to accept a smart parking assistance system and more specifically younger ones. Furthermore, the actual time that the driver usually spends to find a free parking space has a positive impact on the acceptability of the system. The interaction effect indicates that this effect is greater for people who are willing to walk longer from the parking to their final destination and is lower for those who usually park in a public parking area.

Moreover, drivers who are willing to either drive longer in order to find a free parking space, or walk longer from this point to their final destination are not willing to use the proposed system. The willingness to use the app is even lower when the application provides low-certainty feedback and recommendations. On the other hand, if the certainty of the system's recommendation about the parking availability is greater those who usually spend more time when searching for a parking space are more likely to use the application.

Table 2: Estimates of the Logistic Regression model of the acceptance of the application.

\begin{tabular}{|c|c|c|c|}
\hline Variables & Estimate & $\operatorname{Pr}(>|\mathbf{t}|)$ & Sign. $^{1}$ \\
\hline Private parking areas & -0.846 & 0.007 & $* *$ \\
\hline $\begin{array}{l}\text { Gender } \\
(\text { Male }=0 / \text { Female }=1)\end{array}$ & -0.228 & 0.076 & . \\
\hline $\begin{array}{l}\text { Actual time } \\
\text { searching for parking } \\
* \text { Max walking } \\
\text { distance }\end{array}$ & -0.141 & 0.030 & $*$ \\
\hline $\begin{array}{l}\text { Max walking } \\
\text { distance * Maximum } \\
\text { distance willing to } \\
\text { travel }\end{array}$ & 0.200 & 0.001 & $* * *$ \\
\hline $\begin{array}{l}\text { Actual time } \\
\text { searching for parking } \\
* \text { Certainty }\end{array}$ & 0.083 & 0.05 & $*$ \\
\hline $\begin{array}{l}\text { Maximum distance } \\
\text { willing to travel * } \\
\text { Certainty }\end{array}$ & -0.134 & 0.001 & $* *$ \\
\hline $\begin{array}{l}\text { Area* Maximum } \\
\text { distance willing to } \\
\text { travel }\end{array}$ & -0.078 & 0.048 & $*$ \\
\hline $\begin{array}{l}\text { Internet access* } \\
\text { Frequency of detour }\end{array}$ & -0.085 & 0.006 & $* *$ \\
\hline $\begin{array}{l}\text { Internet access * } \\
\text { Goods \& Services }\end{array}$ & 0.198 & 0.001 & $* *$ \\
\hline Internet access * Age & -0.172 & 0.000 & $* * *$ \\
\hline Gender $*$ Age & 0.099 & 0.07 & . \\
\hline $\begin{array}{l}\text { Public parking areas } \\
* \text { Actual time } \\
\text { searching for parking }\end{array}$ & -0.239 & 0.09 & • \\
\hline $\begin{array}{l}\text { Private parking areas } \\
* \text { Actual time } \\
\text { searching for parking }\end{array}$ & 0.384 & 0.03 & $*$ \\
\hline $\begin{array}{l}\text { Private parking areas } \\
\text { * Area }\end{array}$ & 0.186 & 0.033 & * \\
\hline
\end{tabular}

Additionally, people who are willing to travel large distances in order to find a free parking space are less likely to use such an assistance system. The impact of this factor is lower when the city where they live is larger.

Finally, the level of modernization of the city and the familiarity of its population with the internet and other smartphone applications is of great 
importance for the acceptance of smart assistance systems as the one proposed. More specifically, people who live in cities where the majority of the population has access to the internet are willing to use the proposed application. The impact of this factor is greater when most of the population uses goods and services through the internet and is lower for the elderly.

\section{CONCLUSIONS}

In this paper, we investigated the factors that affect the acceptance of smart parking assistance systems. The analyses were conducted on data collected through a questionnaire survey, which referred to a real world parking collection data scheme and associated assistance services. The answers of over 500 respondents were modelled using a genetically optimized Logistic Regression model, which was trained to predict the probability of accepting the specific parking assistance system by taking into account a large set of predictors and their interactions.

Results indicate that the proposed application would be useful for people who are not willing to spend too much time in order to find an available parking space as well as to those who are not willing to walk long distances from the parking place to their final destination. Furthermore, findings revealed that the certainty of the provided recommendation significantly influences the effect of the other parameters on the acceptability of the application. Finally, results show that younger males are more likely to use such an application.

The above findings may provide a comprehensive view on the characteristics of potential users of the proposed system. Nevertheless, future research should focus on a more in-depth analysis of users parking behaviour and city's attributes on the acceptance of such a system. Finally, further research should also investigate the characteristics of the users who are willing to pay for such a system.

\section{REFERENCES}

Ahangari, S., Chavis, C., Jeihani, M., \& Moghaddam, Z. R. (2018). Quantifying the Effect of On-Street Parking Information on Congestion Mitigation using a Driving Simulator. Transportation Research Record, 0361198118773893.

Arnott, R., \& Rowse, J. (1999). Modeling Parking. Journal of Urban Economics, 45(1), pp. 97-124.
Arnott, R., \& Inci E. (2006). An Integrated Model of Downtown Parking and Traffic Congestion. Journal of Urban Economics, 60(3), pp.418-442.

Burnham, K. P., \& Anderson, D. R. (2003). Model selection and multimodel inference: a practical informationtheoretic approach. Springer Science \& Business Media. Second edition. Springer: New York.

Calcagno, V. \& de Mazancourt, C. (2010). glmulti: an R package for easy automated model selection with (generalized) linear models. Journal of statistical software, 34(12), pp. 1-29.

Chatman, D. G. \& Manville, M. (2014). Theory Versus Implementation in Congestion-priced Parking: An Evaluation of SFpark, 2011-2012. Research in Transportation Economics, 44, pp. 52-60.

D’Aloia, M., Rizzi, M., Russo, R., Notarnicola, M. N. \& Pellicani, L. (2015). A marker-based image processing method for detecting available parking slots from UAVs, New Trends Image Analysis Processing. Cham, Switzerland: Springer, pp. 275-281.

Faheem, F., Mahmud, S.A., Khan, G.M., Rahman, M. \& Zafar, H. (2013) A Survey of Intelligent Car Parking System, Journal of Applied Research and Technology, 11(5), pp. 714-726.

Fraifer, M., \& Fernström, M. (2016). Investigation of smart parking systems and their technologies. $37^{\text {th }}$ International Conference on Information Systems. IoT Smart City Challenges Applications (ISCA 2016), Dublin, Ireland, pp. 1-14.

Golias, K. \& Vlahogianni, E. I. (2018). Convolutional Neural Networks for On-Street Parking Space Detection in Urban Networks. IEEE Transactions on Intelligent Transportation Systems.

Ibeas, A., dell'Olio, L., Bordagaray, M. \& Ortúzar, J.D. (2014). Modelling parking choices considering user heterogeneity. Transportation Research Part A: Policy and Practice, 70, pp. 41-49.

Lin, T., Rivano, H., Le Mouël, F., Baron, B., Spathis, P., Rivano, H. \& Voge, M. E. (2017). A survey of smart parking solutions, IEEE Transactions on Intelligent Transportation Systems, 18(12), pp. 3229-3253.

Margreiter, M., Mayer, P. \& Orfanou, F. (2015). A Concept for Crowdsourcing of In-vehicle Data to Improve Urban On-street Parking, mobil.TUM International Scientific Conference on Mobility and Transport "Technologies, Solutions and Perspectives for Intelligent Transport Systems", June 2015.

Margreiter, M., Orfanou, F. \& Mayer, P. (2017). Determination of the parking place availability using manual data collection enriched by crowdsourced invehicle data, Transportation research procedia, $25, \mathrm{pp}$. 497-510.

Monteiro, F.V. \& Ioannou, P. (2018) On-Street Parking Prediction Using Real-Time Data, $21^{\text {st }}$ International Conference on Intelligent Transportation Systems (ITSC), November 4-7, Maui, Hawaii, USA.

Orfanou, F., Margreiter, M., Mayer, Ph., Alpas, M. \& Vlahogianni E. (2017). Driver's Willingness to Use Parking Assistance Tools and their Expectations: A Case Study for the Cities of Munich and Athens, $8^{\text {th }}$ International Congress on Transportation Research in 
Greece "Transportation by 2030: Trends and Perspectives", September Thessaloniki, Greece.

Revathi, G., \& Dhulipala, V. S. (2012). Smart parking systems and sensors: A survey. Computing, Communication and Applications (ICCCA), 2012 International Conference, pp. 1-5, IEEE.

Shiftan, Y., \& Burd-Eden, R. Modeling Response to Parking Policy (2001). Transportation Research Record: Journal of the Transportation Research Board, 1765, pp. 27-34.

Shoup, D. C. (2006). Cruising for Parking. Transport Policy, 13(6), pp. 479-486.

Soto, J. J., Márquez, L., \& Macea, L. F. (2018). Accounting for attitudes on parking choice: An integrated choice and latent variable approach. Transportation Research Part A: Policy and Practice, 111, pp. 65-77.

Van Ommeren, J., Wentink, D. \& Dekkers, J. (2011). The Real Price of Parking Policy. Journal of Urban Economics, 70(1), pp. 25-31.

Vlahogianni, E. I., Karlaftis, M. G., \& Golias, J. C. (2014) Short-term traffic forecasting: Where we are and where we're going. Transportation Research Part $C$ : Emerging Technologies, 43, 3-19.

Vlahogianni, E. I., Kepaptsoglou, K., Tsetsos, V. \& Karlaftis, M.G. (2015). A Real-Time Parking Prediction System for Smart Cities, Journal of Intelligent Transportation Systems, 2450(November), pp. 1-13.

Worldsensing (2019). [Online]. Available at: http://www.fastprk.com/ [Accessed: 3 Jan. 2019]

Yang, W.X. (2004). An Improved Genetic Algorithm Adopting Immigration Operator. Intelligent Data Analysis, 8, pp. 385-401. 